\title{
Literature.
}

Full references to the literature of celestial mechanics are given in the following works :

Gautier: "Essai historique sur le problème des trois corps." Paris, 1817.

Cayley : "On the progress of the solution of certain problems in dynamics." B. A. Reports, 1862.

Dziobek: Die mathematischen Theorien der PlanetenBewegungen. Leipzig, 1888.

Tisserand: Traité de mécanique céleste. Paris, vol. 1, 1889 ; vol. 2 , 1891 ; vol. 3 , 1894 ; vol. $4,1896$.

Brown : Introductory treatise on the lunar theory. Cambridge, 1896.

Whittaker: "Report on the progress of the solution of the problem of three bodies. B. A. Reports, 1899.

Wislicenus: Astronomischer Jahresbericht. Berlin, vol. 1 for 1899 , vol. 2 for 1900 .

It may be added that most of Gylden's work is to be found in the volumes of the Acta Mathematica and in the "Traite analytique des orbites absolues des huit planètes principales" (Stockholm, vol. 1, 1893).

The treatise of Poincaré, frequently referred to above, has been mainly used in the preparation of these lectures. It consists of three volumes published in $1892,1893,1899$, respectively, and is an expansion of the memoir in vol. 13 (1890) of the Acta Mathematica entitled, "Sur le problème des trois corps et les équations de la dynamique." Most of this author's other papers on these subjects will be found in the volumes of the Bulletin Astronomique.

\section{THE HAMBURG MEETING OF THE DEUTSCHE MATHEMATIKER-VEREINIGUNG, SEPTEMBER, 1901.}

The annual meeting of the Deutsche Mathematiker-Vereinigung was held at Hamburg, September 22-28. In the absence of the president of the society, Professor von Dyck, Professor Hilbert occupied the chair. At the business meeting Professor Gutzmer reported on a plan for changing the Jahresbericht into a monthly periodical to be the official organ of the society, and to contain mathematical papers, univer- 
sity and personal news, accounts of the meetings of other mathematical societies, and similar items of general interest. The plan was adopted and Professor Gutzmer elected editor. The financial management will be conducted by the Teubner publishing firm.

The following papers were read at the meeting :

Professor SchouTe, Groningen : "On the mobility of a nullsystem $N_{2 n-1}$ in $R_{2 n-1}$."

Professor ScHubERT, Hamburg: "On the number of constants of the $n$-dimensional generalization of polyedra."

Dr. JAHNKE, Berlin: "On rotations in four dimensional space."

Professor Study, Greifswald: "A new' branch of geometry.",

Professors KLein, Göttingen ; Meyer, Koenigsberg ; SomMERFELD, Aachen : "An account of the present condition of the Encyklopädie der mathematischen Wissenschaften."

Professor von Lilienthal, Münster : "The geometry of motion in its application to differential geometry."

Professor STÄCKEL, Kiel : "Arithmetical characteristics of analytical functions."

Professor Eberhard, Halle: "Contribution to the theory of equations."

Professor StÄckel, Kiel : "Report on the development of instruction in applied mathematics in the German universities.'

Professor Engel, Leipsic: "Higher differential quotients."

Professor Meyer, Koenigsberg: "The extension of the theorems of Henrici and Ivory"

Professor Mittag-Leffler, Stockholm : "A criterion for finding the singularities of analytical functions."

Herr TonkA, Berlin : "On a new geometry of motion."

Professor Schrlling, Göttingen:" "New kinematical models in tooth gear and their relation to the theory of contact transformations."

Professor HAUK, Charlottenburg: "The relations between three parallel projections of a space system."

Professor ADLER, Prague: "Spherical representation of surfaces and its application in descriptive geometry."'

Professor MÜLLER, Königsberg: "On the analogue to Lie's spherical geometry in the domain of the straight line."

Professor London, Breslau : "On a particular kind of converging point series.'

Professor Hilbert, Göttingen : "On certain recent mathematical dissertations." 
Dr. Zermelo, Göttingen : " On the theory of the shortest lines."

Papers on astronomy and geodesy were read by Professor Charlier, Lund; Dr. Halm, Edinburgh ; Dr. Hartwig, Hamburg; Dr. Marcuse, Berlin ; Dr. Ebert, Kiel.

In Professor Schoute's paper the nullsystem was developed analytically and geometrically for higher space (necessarily of an odd number of dimensions). The motion under which the nullsystem remains invariant in space of $2 n-1$ dimensions is the most general motion of a rigid body in that space-a translation and $n-1$ rotations. The relation of the nullsystem to mechanics was mentioned.

Professor Schubert showed that the number of constants of the $n$ dimensional polyedra could not in general be expressed by means of the number of constituent elements of lower dimensions. The number of constants of the $n$ dimensional tetrahedron, hexahedron, and octahedron was developed as $n(n+1), 2 n^{2}$, and $2 n^{2}$, respectively, for $n>4$.

Dr. Jahnke expressed the rotations in four dimensional space by the parameters Euler used to represent the direction cosines of an orthogonal system and showed the relation existing between a four dimensional rotation and two correlated three dimensional rotations. The results find application to the motion of a body in an ideal liquid and to the rotation about its center of gravity of a body carrying an axis about which a second body rotates.

In Professor Study's paper a new line geometry was developed. In the ordinary line geometry the $\infty^{2}$ ideal (uneigentlich) or infinitely distant lines are adjoined to the $\infty^{4}$ actual (eigentlich) lines to form a closed continuum as defined by Cantor. This continuum can be represented continuously on a quadratic manifold in a plane space of five dimensions. The collineations are determinate, single valued, and continuous.

The manifold of the $\infty^{4}$ actual lines can, however, be amplified to a continuum in an entirely different way. Professor Study adjoins the $\infty^{2}$ infinitely distant points to the $\infty^{4}$ actual lines. This manifold forms a closed continuum which can be represented continuously on a manifold of sixth order in a plane space of eight dimensions. There exists a continuous group with 17 parameters whose transformations are determinate, single valued, and continu- 
ous, and the invariant configuration of this group is that of an actual line with all other lines which cut it at right angles. These transformations define a new geometry which may be compared to the Plücker line geometry, but which differs radically from it, as was shown by several theorems which Professor Study stated.

Professors Klein, Meyer, and Sommerfeld reported on the progress of the "Encyklopädie der mathematischen Wissenschaften." 'The difficulties in its preparation are much greater than at first supposed, but they are being steadily overcome, and the work is progressing satisfactorily in all directions.

Professor von Lilienthal mentioned the twists studied by Beltrami in connection with curvature of curves, and then considered the infinitesimal displacement of a rectangular trihedron, one edge coinciding with the normal to a given surface at a point $P$, and the two others with tangents to two curves $C_{1}$ and $C_{2}$ of a given orthogonal family, as $P$ moves on the surface. The intersections of the tangent plane to the surface at $P$ with the axes of the linear family of twists which produce the displacement, lie on a straight line $L$ which passes through the middle points of geodetic curvature of $C_{1}$ and $C_{2}$. Let the principal radii of curvature at $P$ be $\rho_{1}$ and $\rho_{2}$, and the points of intersection of the line $L$ with the principal tangents at $P$ be $P_{2}$ and $P_{1}$ respectively. If $\overline{P P_{1}}=l_{1}, \overline{P P}_{2}=l_{2}$, and if we write $K_{1}$ and $K_{2}$ for the radii of geodetic curvature of $C_{1}$ and $C_{2}$, and $\varphi$ for the angle between $C_{1}$ and the line of curvature corresponding to $\rho_{1}$, then

$$
\frac{1}{K_{1}}=\frac{\cos \varphi}{l_{2}}-\frac{\sin \varphi}{l_{1}}, \quad \frac{1}{K_{2}}=\frac{\cos \varphi}{l_{2}}+\frac{\sin \varphi}{l_{1}},
$$

an expression for the geodetic curvature similar to that of Euler for the curvature of a normal section. Comparing these expressions with those of Liouville for $K_{1}$ and $K_{2}$ we have a geometrical condition for the derivatives of $\varphi$ with respect to the arcs of the lines of curvature, namely,

$$
\frac{1}{l_{1}}=\frac{1}{R_{1}}+\frac{d \varphi}{d s_{1}}, \quad \frac{1}{l_{2}}=\frac{1}{R_{2}}-\frac{d \varphi}{d s_{2}}
$$

where $R_{1}$ and $R_{2}$ are the radii of geodetic curvature of the lines of curvature. It was shown that the axes of the 
above mentioned twists form a cylindroid, the properties of which were considered.

Professor Stäckel's paper was an account of his investigations in continuation of those published by him in the $A n$ nalen and in the Comptes rendus. In attempting to characterize the algebraic functions arithmetically, Professor Stäckel finds that an analytical function $p(x, y)$ is not necessarily algebraic when it is assumed that, in the equation $p(x, y)$ $=0$, to every algebraic $x$ there corresponds an algebraic $y$, and to every algebraic $y$ there corresponds an algebraic $x$. An arithmetical criterion for algebraic functions has not yet been found. The following theorem was proved: When for a single valued analytical function, having its one essential singularity at infinity, and possessing an algebraic addition theorem, such a point exists that the coefficients in the development of the function in a power series about the point are all algebraic numbers, then the function can be simply periodic only when the period is a transcendental number. In conclusion Professor Stäckel investigated the nature of the periods of such elliptic functions as admit of complex multiplication.

The object of Professor Eberhard's paper was to find the number and approximate the position of the real roots of an algebraic equation with real coefficients. Let this equation, of degree $n$, be $f(x)=0$. Professor Eberhard builds two auxiliary equations of degree at most $n-1$, namely,

$$
\frac{d}{d x} f(x)=0, \quad\left[\frac{d}{d x^{\prime}} f\left(\frac{1}{x^{\prime}}\right)\right]_{x^{\prime}=\frac{1}{x}}=0,
$$

$i$. e., the derived equation, and the reciprocal of the derived equation of the reciprocal. If the real roots $\alpha_{i}, \beta_{i}$ of these two equations be arranged in one series according to magnitude, then the position of the real roots of $f(x)=0$ can be found relative to the terms of this series, $i$. e., relative to the roots $\alpha_{i}, \beta_{i}$, of equations of lower degree.

Professor Stäckel gave, in his second paper, an account of the development in instruction in applied mathematics at the German universities. With the founding of technical schools the study of applied mathematics at the universities lost strength, and only in the last few years, and largely through the efforts of Professor Klein, has the work begun to assume a place of importance. The universities of Göt- 
tingen and Strassburg were the first to offer regular and extended courses in descriptive geometry, geodesy, and technical mechanics, and their lead is being rapidly followed by the other institutions.

The object of Professor Engel's paper was the generalization of the Clebsch " Konnexcoördinaten " for the geometry of differential equations of order higher than the first. In the Leipziger Berichte, 1893, Professor Engel showed how this could be accomplished, but the complicated form of the result prevented its practical application. Professor Study (Leipziger Berichte, 1901) has solved the problem for a differential equation of second order in the plane, and Professor Engel gave in the present paper the corresponding development for partial differential equations of second order in space.

Professor Meyer's paper dealt with the generalization of the theorems of Henrici and Ivory. If $P_{1}, P_{2}$ be two points on a quadric surface, $F_{1}$, and if these points go into $P_{1}^{\prime}, P_{2}^{\prime}$ by the transformation which carries $F_{1}$ into any confocal quadric $F_{2}$, then in general (Ivory's theorem) $\overline{P_{1} P_{2}^{\prime}}=$ $\overline{P_{2} P_{1}^{\prime}}$; and if $P_{1}$ and $P_{2}$ lie on a generating line of $F_{1}$, then (Henrici's theorem) $\bar{P}_{1} P_{2}=\overline{P_{1}^{\prime} P_{2}^{\prime}}$. Professor Meyer considered the algebraic identities which express these theorems and proved that each theorem was characteristic for confocal quadrics. If the ratio of the distances be assumed to be any constant, then the quadrics $F_{1}$ and $F_{2}$ must be similar and similarly placed. A generalization to pairs of coaxial quadrics was also made.

The generalization to surfaces of higher order which have a conic at infinity is especially interesting. Consider a surface of fourth order which has the conic $C_{2}$ at infinity. If $O$ be any point in space, and $O_{1}, O_{2}, O_{3}$ the principal axes of the cone from $O$ to $C_{2}$, then there exists an $\infty^{1}$ family of affine transformations $A$ with the principal axes $O_{1}, O_{2}, O_{3}$, of such a nature that the distance between any two points $P_{1}, P_{2}$ of the surface of fourth order, whose joining line meets the surface at infinity, is invariant with respect to the transformations $A$. Similar theorems hold for surfaces of order $n$.

The position of the theorems of Henrici and Ivory in the theory of quadric surfaces is made more clear by the following considerations. Let the points $P_{1}, P_{2}$ have any position in space. The distance $\overline{P_{1} P_{2}}$ remains invariant under a six parameter group of collineations. If however $P_{1}, P_{2}$ 
be restricted to lie on a generating line of a fixed quadric, then the six parameter group is extended to a seven parameter group by the addition of the above mentioned $\infty^{1}$ family of affine transformations $A$. The case of Ivory's theorem is similar; the collineations which change every line $P_{1} P_{2}$ into $P_{1}^{\prime} P_{2}^{\prime}$ in such a way that $\overline{P_{1} P_{2}^{\prime}}=\overline{P_{2} P_{1}^{\prime}}$ form a two parameter family $S$ of "symmetric rotations." If $P_{1}, P_{2}$ lie on a fixed quadric, then the $\infty^{1}$ family of affine transformations $A$ is added to $S$ to form a three parameter group of collineations under which $\overline{P_{1} P_{2}^{\prime}}=\overline{P_{2} P_{1}^{\prime}}$.

Professor Mittag-Leffler spoke on the singularities of analytical functions. Hadamard and Lindelöf have given criteria for single points on the circle of convergence of a power series. The problem may be stated in another way and the analytical continuation of a power series be demanded along a given line. Professor Mittag-Leffler finds how far this is possible by building the necessary and sufficient condition for a singular point on the given line.

Herr Torka's paper dealt with the geometry of motion produced by various links and linkages.

Professor Schilling explained his new and interesting kinematical models for the generation of curves and pointed out their relation to Lie's contact transformations. The paper was illustrated by the working models and by projected diagrams.

Professor Hauk gave the conditions under which projective systems in three planes can be the parallel projections of a single space system, derived the relations which then exist between the elements of this system, and gave various methods of determining it.

Professor Adler's paper dealt with the spherical representation of surfaces. The polar of a given surface with respect to a unit sphere is projected from the center to the surface of the sphere. The lines of curvature of the original. surface go into confocal spherical conics. The spherical representation of the lines of equal illumination of any surface in parallel light are also the lines of equal illumination of the sphere in the same light, and on this theorem rests the application to descriptive geometry.

Professor Müller showed that the projective geometry 
on a straight line forms an analogue to Lie's spherical geometry.

Professor London gave a construction which approximates to the point of convergence of certain point series (Punktfolgen) to any desired degree of precision. Let the points of a line be projectively related. To any initial point $P_{1}$ construct the corresponding point $P_{2}$, to $P_{2}$ again the corresponding point, etc. If the projective relation has real double points and does not form an involution, then the point series formed in the above manner converges to that double point which is furthest from the vanishing point. The point series formed similarly from the inverse projective relation converges to the other double point. If the double points are imaginary the points of the series lie everywhere dense on the line. Similar relations hold in the case of collineations of a plane, and also for three and higher dimensions.

Professor Hilbert spoke on the theses which have been written under his direction at Göttingen during the last two years. These may be divided roughly into three groups : the theses of Reid (1899), Hilbert (1900), Rueckel (1901), Bernstein (1901), and Fräulein v. Saposki (not yet appeared), deal with theory of numbers; those of Hedrick (1901), Noble (1901), J. Müller, and Fräulein v. Gernet (not yet appeared), with the calculus of variations and differential equations, and those of Feldblum (1899), Dehn (1901), Boy (1901), Zoll (Göttingen Preisarbeit, 1901), and Hamel (1901), with geometrical questions. Professor Hilbert spoke in detail on the last group only, and especially on the last three theses.

Herr Boy has succeeded in finding a finite closed surface without singularities whose points have a one to one correspondence to the points of the projective plane, and which has, therefore, the same relation to the projective plane as the sphere to the complex plane. The surface cuts itself but has no pinch points. It is, in a way, the simplest surface next to the sphere, as it has one maximum, one minimum and one saddle point.

Herr Zoll has been able to find closed surfaces without singularities upon which all geodetic lines are closed.

Herr Hamel has studied the geometries in which it is not assumed that the angles at the base of an isosceles triangle are equal, but instead that the straight line is the shortest distance. It is shown that there exist geometries of this 
nature other than the two already found by Minkowski and Hilbert.

Dr. Zermelo's paper treated of three problems in the theory of geodetic lines: the absolute shortest line; the shortest line in a bounded region ; the shortest line with restricted steepness.

A line on a surface is an absolute or relative shortest line, according as it is the shortest of all possible lines on the surface between two given points, or the shortest of all lines that lie in a strip as narrow as we please about it. Consider a point 0 , and an extremal (a line satisfying Lagrange's equation) $L$ through 0 . It is known that the extremal $L$ is the relative shortest line up to its point of tangency 1 , with the envelope of extremals through 0 ; it ceases, however, to be the absolute shortest line before the point 1 is reached. An absolute shortest line $l, l_{1}<L_{1}$, (where the subscript letters denote lengths) must exist* between the points 0 and 1 and must be an extremal through 0 which cuts the extremal $L$ and the envelope at the point 1 . If the parameter in the equation of $l$ be so varied that the point of intersection $\alpha$ of $l$ with $L$ moves from 1 towards 0 , then, from reasons of continuity, we have $l \alpha<L \alpha$, up to a certain point 2 where $l_{2}=L_{2}$. The locus of such points 2 for all extremals through 0 forms a curve which Dr. Fermelo calls the "Doppelabstandscurve." This curve passes in general through cusps of the envelope.

The shortest line in a bounded plane region consists of straight lines and portions of the boundary convex towards the interior. The following theorem was given : In any simply connected plane region, the shortest line between two given points is uniquely determined, and is therefore the absolute shortest.

The third problem is that of shortest lines with restricted steepness, $K=\frac{d z}{d s}<K_{0}$, on the surface $z=\varphi(x, y)$. The shortest lines consist of geodetic lines and lines of constant steepness, $K=K_{0}$. The following theorem was proved : Every curve of greater steepness, $K>K_{0}$, can be replaced by zigzag lines of constant steepness, $K=K_{0}$, which run as near as we please to the curve, and which satisfy the conditions of the variation problem.

I wish to thank the speakers who so kindly responded to my requests for information, and to acknowledge the use

* Proved by Hilbert. See Noble, Diss., Göttingen, 1901. 
which the notes of Dr. Schröder, secretary of the meeting, have been to me.

GöTTINGEN,

C. M. Mason.

October, 1901.

\section{SOME CURIOUS PROPERTIES OF CONICS TOUCH- ING THE LINE INFINITY AT ONE OF THE CIRCULAR POINTS.}

BY K. V. HUNTINGTON AND J. K. WHITTEMORK.

(Read before the American Mathematical Society, October 26, 1901.)

The subject of the present note was suggested by the apparent contradiction between the following well known theorems of modern analytic geometry :

1. Through any four given points of the plane there pass two parabolas.

2. Through the vertices and the orthocenter of a triangle there pass no conics but equilateral hyperbolas.

The difficulty here presented may be removed as follows : We know that conics through four points determine an involution on any line of the plane. Since the conics of theorem 2 are equilateral hyperbolas the involution which they determine on the line infinity will have the circular points $I$ and $J$ as double points. Two of the hyperbolas are therefore tangent to the line infinity, one at $I$ and the other at $J$. But any conic tangent to the line infinity is a parabola ; therefore these two hyperbolas are parabolas, and the contradiction with Theorem 1 disappears.

We shall now investigate these curves more closely.

Let $S$ be any conic touching the line infinity at one of the circular points, $I$.

The conic $S$ is a parabola, being tangent to the line infinity. But the straight lines joining any finite point with the infinite points of the curve are perpendicular, since any line through $I$ is perpendicular to itself ; hence $S$ is also an equilateral hyperbola.

The center of $S, i$. e., the pole of the line infinity, is the point $I$.

The four foci, $i$. e., the points of intersection of tangents from $I$ and $J$, coincide with $J$.

The four directrices coincide with the polar of $J$, and pass therefore through $I$. 